\title{
EVALUACIÓN DE LA PRODUCCIÓN DE SEDIMENTOS Y CALIDAD DE LAS AGUAS EN CUENCAS AGRARIAS DE NAVARRA
}

\author{
R. GIMÉNEZ* , J. CASALÍ, J. DÍEZ \\ Universidad Pública de Navarra, E.T.S. de Ingenieros Agrónomos, \\ Departamento de Proyectos e Ingeniería Rural.
}

RESUMEN. Para evaluar el efecto medioambiental de la actividad agraria, el Gobierno de Navarra dispone de una red de cuencas experimentales donde se registran - en forma continua-datos hidrológicos y meteorológicos y de calidad de las aguas. En este artículo se reseña los resultados obtenidos-luego de cerca de 15 años de registros-en dos cuencas cerealistas -La Tejería y Latxaga-y una ganadera-forestal con una sub-cuenca casi enteramente forestal -Oskotz-, en cuanto a precipitación, escorrentía y exportación de sedimentos, nitratos y fosfatos. Se analiza tanto la variabilidad interanual como la espacial (o intercuencas) del proceso de exportación y sus implicaciones en la calidad del agua y en el ecosistema local.

Aunque las lluvias estivales son las potencialmente más erosivas, el mayor registro de sedimentos en todas las cuencas se produce en invierno. Esto, se atribuye al alto contenido de humedad de los suelos en esta época, lo cual facilita la generación de escorrentía.

Cuencas cerealistas, aparentemente, similares presentan marcadas diferencias en exportación de sedimentos: en La Tejería, la producción anual media es de $1800 \mathrm{~kg} / \mathrm{ha}$ año, mientras que en Latxaga alcanza sólo $450 \mathrm{~kg} / \mathrm{ha}$ año. Las diferencias morfológicas, topográficas y de cobertura vegetal de los cauces entre ambas cuencas explicarían - en gran parte-estas discrepancias. En Oskotz, los valores son normalmente inferiores a los registrados en cuencas cerealistas, pero a veces superiores a los de Latxaga, dependiendo del manejo forestal que se haya hecho.

La calidad del agua se ve seriamente afectada en La Tejería por la polución con nitratos, siempre superior a valores críticos. En Latxaga, en cambio, la contaminación por nitrato es mucho menor. La contaminación por fosfatos supera, en ambas cuencas cerealistas, los umbrales de tolerancia. La actividad ganadera genera, en Oskotz, insospechados niveles de fosfatos, muy superiores a los registrados en las cuencas cerealistas. 


\title{
Evaluation of sediment yield and water quality in agricultural catchments of Navarre (Spain)
}

\begin{abstract}
The Government of Navarre (Spain) established a series of experimental watersheds on different sites of its territory in order to assess the impact of the agricultural activities on the environment. Hydrological/meteorological and water quality data are continuously monitored. In this paper, data recorded -precipitation, runoff, sediment, nitrate and phosphate- in two grain-sown watersheds (La Tejería and Latxaga) and in a third one (Oskotz) covered with forest and pasture (cattle-breeding) with a sub-watershed almost entirely under forest, are analyzed; the information covers a period of ca. 15 years. The inter/intra annual variability of the involved processes are studied as well as their impact on the water quality and on the ecosystem.

The majority of the sediments yielded in the watersheds are registered during winter, though most of the erosive rainfalls occurred in summer. This is because of the easy-prone runoff that prevailed during winter due to the (much) higher water content of the soils in this period.

The grain-sown watersheds -roughly similar at first view- present however important differences regarding sediment yield: $1800 \mathrm{~kg} / \mathrm{ha}$ year at La Tejería and only $450 \mathrm{~kg} / \mathrm{ha}$ year at Latxaga. This could be mainly due to differences in morphology, topography, and amount of stream channel vegetation between both sites. At Oskotz this figures are as a whole lower than those registered in the cultivated watersheds but eventually larger than those at Latxaga; this depending on the forest management (logging).

Water quality is seriously affected at La Tejería with nitrate concentration constantly over critical threshold. By contrast, nitrate values are much lower in Latxaga watershed. However, phosphate concentrations were similar in both grain-sown watersheds corresponding to water with a significant risk of eutrophication. The cattle-breeding activity at Oskotz generated unexpected levels of phosphate much higher than those registered in the cultivated wathersheds.
\end{abstract}

Palabras clave: escorrentía, sedimentos, nitratos, fosfatos, calidad del agua, cuencas experimentales, región mediterránea, uso del suelo, Navarra.

Key words: runoff, sediment, nitrate, phosphate, water quality, experimental watersheds, Mediterranean region, land use, Navarre.

Enviado el 12 de septiembre de 2011

Aceptado el 9 de noviembre de 2011

* Correspondencia: Departamento de Proyectos e Ingeniría Rural. Universidad Pública de Navarra. Edificio Departamental de los Olivos, 31006, Pamplona (España). E-mail: rafael.gimenez@unavarra.es. 


\section{Introducción}

La erosión de los suelos, así como la mayor parte de los procesos geomorfológicos, son escalo-dependientes; de tal manera que con el aumento de la escala espacial se incrementan tanto el número de factores intervinientes en dichos procesos como también la complejidad de su interacción (Morgan, 2005; Boix-Fayos et al., 2006). Por ejemplo, a micro escala $\left(<1 \mathrm{~m}^{2}\right)$, la erosión estará principalmente condicionada por la estabilidad estructural de los agregados del suelo y por la frecuencia y erosividad de las lluvias; mientras que, a meso-escala $\left(1 \mathrm{~m}^{2}-100 \mathrm{~m}^{2}\right)$, la erosión dependerá, además, de los factores condicionantes de la escorrentía como pueden ser la capacidad y variabilidad espacial de la infiltración del suelo, de cambios en la rugosidad y pedregosidad superficial, etc. A partir de un enfoque a macro-escala y especialmente a escala de cuenca $(>10000$ $\mathrm{m}^{2}$ ), debemos considerar también entre los factores condicionantes la combinación de características topográficas - por ej. pendiente y formas de relieve- con los distintos tipos, usos y distribución de suelos. Por otra parte, a medida que la escala aumenta, la natural conectividad entre los distintos factores del sistema hidrológico (Ej., red de drenaje) quedará mejor reflejada o representada (Boix-Fayos et al., 2006). En definitiva, los estudios a nivel de cuencas aportan la posibilidad de estudiar la erosión del suelo y el transporte de sedimentos y solutos desde una perspectiva sistémica; que permite relacionar y jerarquizar la influencia de los diferentes factores que intervienen en los procesos hidrológicos-erosivos.

Concretamente, la investigación a nivel de cuencas permite (i) evaluar la dinámica de sedimentos, (ii) integrar la información hidromorfológica de laderas y cauces, (iii) relacionar episodios pluviométricos e hidrológicos con la actividad geomorfológica, (v) valorar el papel de las diferentes fuentes de sedimento y (vi) analizar los efectos de distintas cubiertas vegetales y de usos del suelo (García Ruiz y López Bermúdez, 2009).

En Navarra, la erosión de los suelos es un importante problema que afecta las tierras agrícolas (Casalí, 1999; De Santisteban et al., 2006; García Ruiz y López Bermúdez, 2009). Precisamente, con el objeto de valorar el efecto de la actividad agraria sobre el entorno y contemplar mejoras en el manejo de las zonas afectadas, el Gobierno de Navarra viene recopilando - desde hace ya varios años-información territorial, meteorológica, de erosión y de calidad de aguas (nitratos, fosfatos y potasio, entre otras substancias), en cuencas agrarias típicas de su territorio. Se pretende que esta red de cuencas experimentales siga en funcionamiento de forma permanente y continua para conseguir adecuadamente los objetivos arriba mencionados; esto es así debido a la elevada variabilidad de los parámetros involucrados, propia de nuestro clima. La información así obtenida es, por otro lado, de gran utilidad para una mejor estimación de los recursos hídricos disponibles, para la caracterización hidrológica de las zonas estudiadas, y para la evaluación de diversos modelos de simulación de interés en la gestión y planificación del territorio.

Concretamente, están en funcionamiento en la actualidad 4 cuencas experimentales. Dos de ellas, denominadas Latxaga y La Tejería ( 200 ha, cada una), representan las condiciones de cultivo de los buenos secanos cerealistas de la zona media de Navarra, mientras que la tercera, Oskotz ( 1600 ha), localizada en la montaña, se encuentra 
sometida a una actividad ganadera intensa. Una cuarta cuenca, perteneciente al río Cemborain, por su mayor tamaño ( 5000 ha) posee un variado uso del suelo: el $25 \%$ destinado a usos agrícolas, el $40 \%$ forestal, $30 \%$ bajo matorral y el resto ocupado por pastizales y terrenos urbanos. De las cuencas de Latxaga y La Tejería se cuenta con datos desde 1996, de Oskotz desde 1999, mientras que Cemborain se incorpora a la red de cuencas en forma completamente operativa recién en el transcurso del presente año.

En este trabajo se analizan y discuten brevemente los principales resultados obtenidos en estos años (hasta 2009 inclusive) de actividad agraria en las tres primeras cuencas citadas (Latxaga, La Tejería y Oskotz), en lo referente a precipitación, escorrentía, y exportación de sedimentos, nitratos y fosfatos. Como se entenderá, la cuenca de Cemborain se excluye del análisis debido a la exigua información que todavía se posee de la misma.

\section{Descripción de las cuencas experimentales}

- Latxaga. La cuenca de Latxaga ocupa 207 ha en terrenos de los términos municipales de Lizoain y Urroz-Villa; esto es, en el centro-este de Navarra (fig. 1A).

Presenta un clima submediterraneo húmedo, con una precipitación anual media de $835 \mathrm{~mm}$, distribuida en 95-100 días de lluvia; y con una temperatura media de $12^{\circ} \mathrm{C}$ (Gobierno de Navarra, 2001).

El área se encuentra sobre arcillas margosas y margas grises de Pamplona (Gobierno de Navarra, 1997). Con respecto a su topografía, los fondos de valle son de pendiente suave $(5-7 \%)$, en contraste con la fuerte inclinación (hasta 30\%) de sus laderas (Tabla 1).

Tabla 1. Algunas características morfológicas de las cuencas experimentales en estudio.

\begin{tabular}{|l|c|c|c|}
\hline & Latxaga & La Tejería & Oskotz \\
\hline Área $\left(\mathrm{km}^{2}\right)$ & 2.07 & 1.69 & 16.74 \\
\hline Perímetro $(\mathrm{km})$ & 6.67 & 5.46 & 53.94 \\
\hline Longitud total del canal $(\mathrm{km})$ & 5.38 & 3.20 & 18.49 \\
\hline Mínima elevación $(\mathrm{m})$ & 504 & 496 & 530 \\
\hline Máxima elevación $(\mathrm{m})$ & 639 & 649 & 924 \\
\hline Pendiente promedio $(\%)$ & 19.3 & 14.8 & 20.3 \\
\hline Pendiente promedio del canal principal $(\%)$ & 12.4 & 14.6 & 13.8 \\
\hline Índice de Gravelius & 1.30 & 1.17 & 3.69 \\
\hline Factor de forma & 0.44 & 0.85 & 1.07 \\
\hline Densidad de drenaje $\left(\mathrm{km} \mathrm{km}^{-2}\right)$ & 2.61 & 1.91 & 1.10 \\
\hline
\end{tabular}


Los suelos predominantes son Paralitic Xerorthent y Fluventic Haloxerept (USDA, Soil Taxonomy, 2006). Los primeros, son suelos someros ( $<0.5 \mathrm{~m}$ de profundidad), con un horizonte superficial de textura franco-arcillo-limosa; ocupando el $43 \%$ de la cuenca, principalmente las laderas de erosión. Mientras que los segundos son suelos profundos ( $>1 \mathrm{~m}$ de profundidad) pero con un horizonte superficial de similares características texturales que las del anterior. Normalmente se encuentran en las vaguadas y zonas de deposición.

La cuenca se encuentra ocupada prácticamente en su totalidad (80-90\%) con cultivos de cereales de invierno -trigo, cebada y avena-, con producciones medias de 3500 a $4000 \mathrm{~kg} / \mathrm{ha}$ en las laderas y de $5500 \mathrm{~kg} / \mathrm{ha}$, y superiores, en los suelos más profundos. Los cauces presentan abundante vegetación tanto en sus orillas como en el lecho.

Se practica un laboreo convencional, normalmente siguiendo curvas de nivel. Sin embargo, una (densa) faja de vegetación natural es mantenida a lo largo de los principales cauces.

En cuanto a fertilización -asumiendo que se siguen las pautas del Instituto Técnico de Gestión Agrícola- se aplican unas 230 UFN/ha año; en dos aplicaciones en cobertera, la primera hacia el 15 de enero y la segunda hacia el 15 de marzo. Adicionalmente, cada tres años e inmediatamente antes de la siembra, suele aplicarse 1 UFP por cada 100 $\mathrm{kg}$ de cosecha, lo que equivale a unos $165 \mathrm{~kg}$ DAP (18-46-0) /ha año.

- La Tejería. La cuenca de La Tejería se localiza igualmente en el centro-este de Navarra, pero en el término municipal de Villanueva de Yerri (fig. 1B). La cuenca vierte directamente al embalse de Alloz (de $62.8 \mathrm{Hm}^{3}$ de capacidad), utilizado para riego.

El clima predominante es -al igual que Latxaga- submediterráneo húmedo, pero con una precipitación media algo inferior $(725 \mathrm{~mm})$, distribuida en más de 105 días de lluvia (Gobierno de Navarra, 2001). La temperatura anual media es de $13^{\circ} \mathrm{C}$.

La cuenca se encuentra sobre margas y areniscas de facies continentales (Gobierno de Navarra, 1997). Sus pendientes son muy homogéneas y relativamente suaves, 15\% en promedio (Tabla 1).

En cuanto a sus suelos, la clase predominante es Vertic Haploxerept (USDA, Soil Taxonomy, 2006); ocupando un $41 \%$ de la cuenca, especialmente sobre las laderas de erosión. Son en general suelos relativamente someros (0.5-1 $\mathrm{m}$ de profundidad) y con un horizonte superior de textura limo-arcillosa.

En general, tanto el uso de la tierra, prácticas de manejo del suelo y rendimiento de los cultivos, son similares a los ya descriptos para la cuenca de Latxaga. Igualmente, más del $90 \%$ de la superficie total se dedica al cultivo de cereales. En contraposición, si bien la fertilización es similar a la efectuada en Latxaga, la cantidad total de nitrógeno aplicada es significativamente menor (150 kg N/ha). Además, a diferencia de Latxaga, se destaca la escasa o nula presencia de vegetación en taludes y lecho de los cauces. 

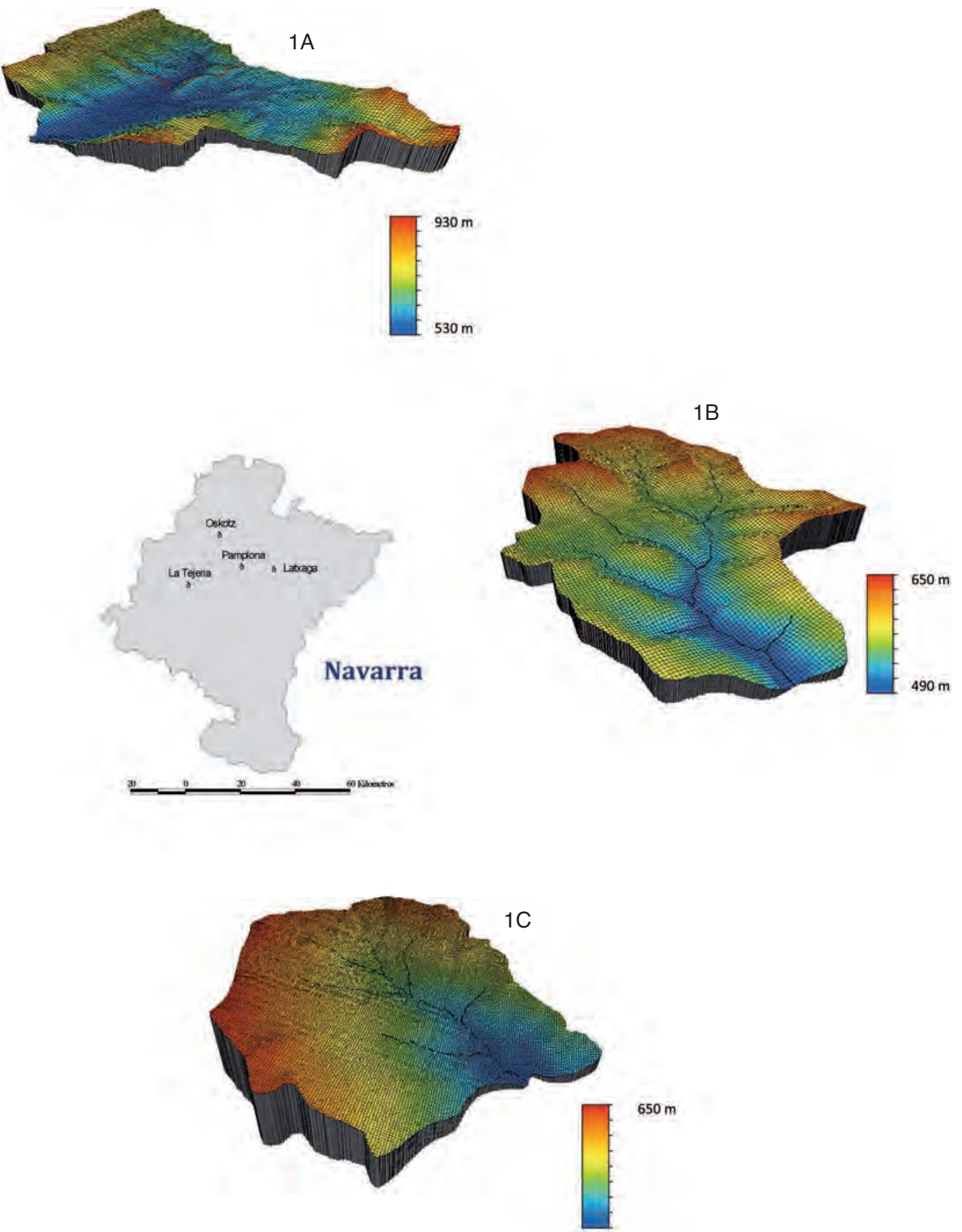

Figura 1. Localización de las cuencas experimentales pertenecientes al Gobierno de Navarra. (1A: Latxaga; 1B: La Tejería; 1C: Oskotz). 
- Oskotz. La cuenca de Oskotz está situada en la zona noroeste de la Comunidad Foral de Navarra, abarcando 1674 ha (fig. 1C). Su clima es subatlántico, con una precipitación media de $1200 \mathrm{~mm}$, distribuida en 130 días de lluvia; mientras que su temperatura media anual alcanza los $12^{\circ} \mathrm{C}$ (Gobierno de Navarra, 2001).

En líneas generales, la geología y la topografía de esta cuenca es similar a la de Latxaga, con pendientes suaves $(5 \%)$ en los fondos llanos del valle y moderadas en las laderas. Sin embargo, en estas últimas, las pendientes pueden llegar a ser considerablemente superiores (hasta 65\%) a las de Latxaga (Tabla 1).

En las laderas sujetas a erosión predominan los suelos Lythic y Typic Ustochrepts (USDA, Soil Taxonomy, 2006); mientras que en el valle, el suelo Fluventic Ustochrepts (USDA, Soil Taxonomy, 2006) es el dominante. Los principales suelos son de clases texturales finas y normalmente de más de $1 \mathrm{~m}$ de profundidad, excepto aquellos ubicados en las laderas erosionadas que presentan un espesor reducido $(0.5-1 \mathrm{~m})$.

En esta cuenca, dos son los usos predominantes de la tierra: más de un $60 \%$ de su superficie está ocupada por bosques con especies como Fagus sylvatica, Quercus pirenaica y Pinus $s p$. Mientras que el resto está cubierto por praderas y pastizales, donde se desarrolla una intensa actividad ganadera (bovinos y ovinos); además de pequeños sectores dedicados a cultivos.

Las pasturas son tanto naturales como cultivadas con un rendimiento medio de aproximadamente $12000 \mathrm{~kg} / \mathrm{ha}$, suma de dos cortes al año. Las primeras -Lolium, Festuca Brachypodium, Bromus, Trifolium y Melitotus- ocupan los suelos más someros y las laderas empinadas. En cuanto a las segundas son sembradas cada 4-5 años, comprendiendo especies como Lolium perenne, Lolium multiflorum, Trifolium pratense y Trifolium repens.

Las pasturas son fertilizadas en mayo con $150 \mathrm{~kg} / \mathrm{ha}$ de urea y en noviembre con una mezcla de P-K-Mg-Zn, a razón de $450 \mathrm{Kg} / \mathrm{ha}$. Además -normalmente en abril-, se realiza una aplicación de purines que aportan aproximadamente $13 \mathrm{~kg} / \mathrm{ha}$ y $22 \mathrm{~kg} / \mathrm{ha}$ de $\mathrm{P}$ y $\mathrm{N}$, respectivamente.

\section{Instrumentación y mediciones en las cuencas}

Cada cuenca está provista de una completa estación meteorológica; y de una estación hidrológica localizada en su salida (Casalí et al., 2008, 2010). En realidad, la cuenca de Oskotz está provista de dos estaciones hidrológicas, una que realiza mediciones de la cuenca en su conjunto (bosques y pastizales), a la que llamaremos "principal", Op. Y una segunda que se localiza a la salida de una subcuenca ( $\sim 500 \mathrm{ha})$ de uso casi exclusivamente forestal, a la que denominaremos por tanto, "forestal", Of.

En cada estación hidrológica se llevan registros, cada 10 minutos, tanto del nivel de agua como de su turbidez. La descarga se determina a partir del nivel del agua, previa calibración realizada a través de mediciones directas con molinetes y vertederos. 
Muestras de agua y sedimento se toman automáticamente cada 6 horas de una cavidad de $0.6 \mathrm{~m}$ de diámetro, practicada ad hoc aguas debajo de la cresta del vertedero. Las 4 muestras diarias se mezclan antes de su análisis (ver abajo), obteniéndose así una única muestra, promedio diario (Isidoro et al., 2003).

El análisis de las muestras se realiza siguiendo el protocolo establecido por el Laboratorio de Agricultura dependiente del Gobierno de Navarra. Concretamente, se determinan concentraciones de sedimentos, nitratos y fosfatos; como así también la de otros solutos no tratados en el presente trabajo.

Cabe destacar que, debido a un mal funcionamiento de los instrumentos, no se cuenta en La Tejería con registros de descarga entre septiembre de 1998 y marzo de 2000; tampoco en Latxaga, entre mayo de 1999 y febrero de 2002. Sin embargo, los pocos datos pluviométricos faltantes fueron estimados $\left(\mathrm{r}^{2}=0.98\right)$ a partir del método propuesto por Llamas (1993), y con datos de estaciones meteorológicas vecinas.

\section{Análisis de datos}

\subsection{Precipitación y escorrentía}

Los valores acumulados medios de precipitación en Latxaga $(830 \mathrm{~mm})$ y La Tejería $(724 \mathrm{~mm})$ son similares entre sí, pero marcadamente inferiores a los registrados en Oskotz (1137 mm) (fig. 2); lo que es de esperar dado el clima más húmedo propio de esta última cuenca. Estas diferencias son igualmente ostensibles en aquellos años de mínimo registro de precipitaciones donde estas cifras son aproximadamente $500 \mathrm{~mm}$ y 750 $\mathrm{mm}$ en las cuencas cerealistas y forestal -Op/Of-, respectivamente. Sin embargo, en años de máxima precipitación los registros medios anuales de las tres cuencas son menos contrastantes: alrededor de $1000 \mathrm{~mm}$ y $1200 \mathrm{~mm}$, respectivamente. Cabe destacar que no se establecen diferencias entre las lluvias acaecidas en Op y Of puesto que cuenca y subcuenca comparten pluviógrafo.

En todas las cuencas se observa un patrón similar de variación de la lluvia a lo largo del año. Esto es, una ligera estacionalidad; con otoños e inviernos húmedos y veranos secos. Aun así, en estos últimos puede registrarse aproximadamente el $15 \%$ del total de las lluvias anuales (fig. 3).

Aunque el mayor registro de precipitaciones se presente en invierno, las lluvias de mayor poder erosivo ocurren en verano. Es así que, si caracterizamos el poder erosivo de las lluvias en las diferentes cuencas a través del índice de erosividad EI30 (Morgan, 2005), se observa que (datos no mostrados) un 70\%-80\% del total de eventos -alrededor de 1000 eventos/cuenca- presentaron una (muy) baja erosividad (EI30<100 J mm $\left.\mathrm{m}^{-2} \mathrm{~h}^{-1}\right)$; mientras que unos pocos eventos -normalmente estivales- presentaron un potencialmente elevado poder erosivo (el valor EI30 se incrementa en 2-3 órdenes de magnitud). Cabe destacar que la erosividad de las precipitaciones -similar en ambas cuencas cerealistas- es, no obstante, marcadamente superior en la cuenca de Oskotz. 

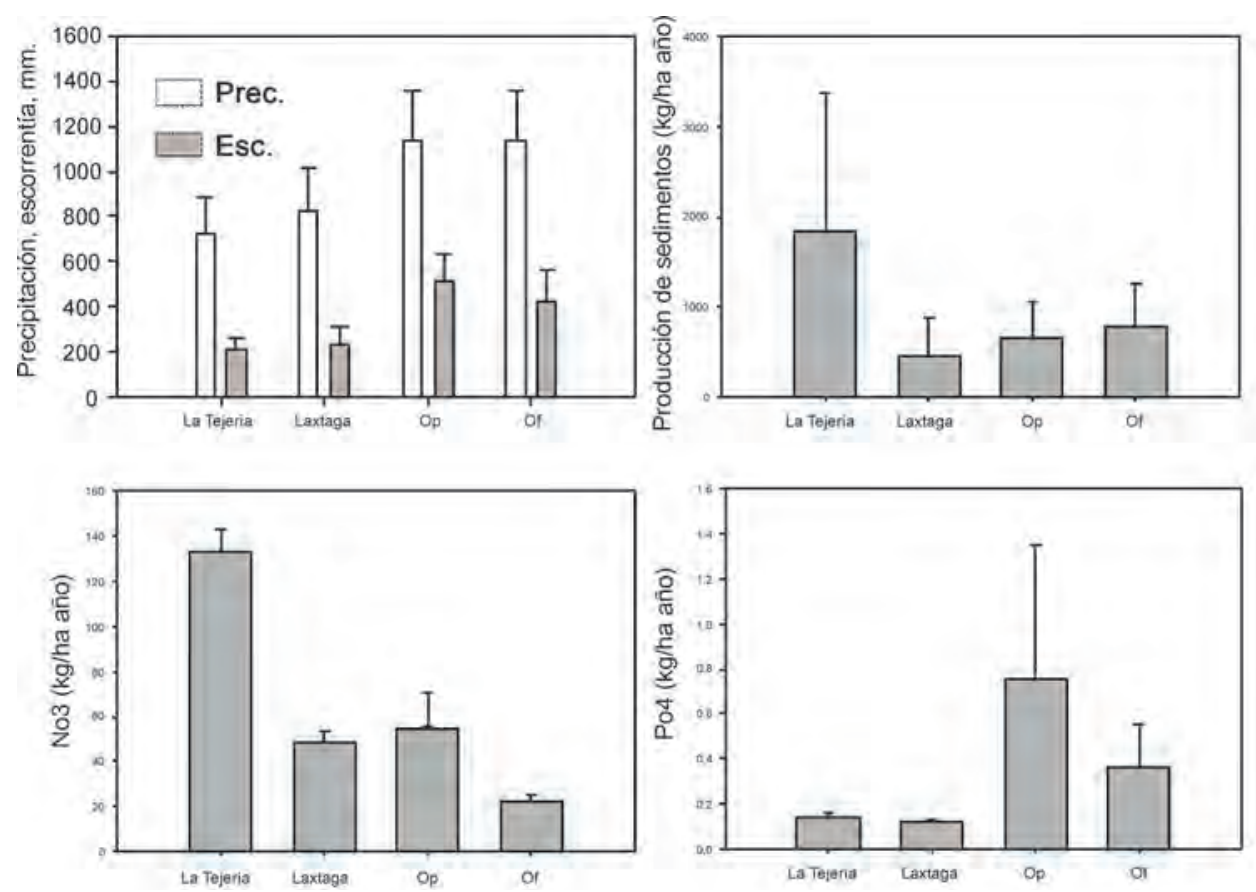

Figura 2. Valores anuales medios de precipitación, escorrentía, descarga de sedimentos, nitratos y fosfatos en las cuencas de La Tejería, Latxaga, Oskotz principal (Op) y Oskotz. forestal (Of). Los valores de nitratos y fosfatos están referidos al área total de cada cuenca. Las barras verticales indican la desviación estándar.

Aun así, y como se discutirá luego, el poder erosivo de la lluvia no basta para explicar cabalmente los registros anuales de sedimentos en las diferentes cuencas.

Con respecto a la escorrentía -y en concordancia con las precipitaciones-, los valores anuales promedios en Latxaga $(234 \mathrm{~mm})$ y La Tejería $(211 \mathrm{~mm})$ son notoriamente inferiores a los registrados en la cuenca de Oskotz (Op: 516 mm, Of: $421 \mathrm{~mm}$ ) (fig. 2). Por otro lado, -y al igual también que en las precipitaciones- se observa una estacionalidad en los registros de escorrentía; pero esta estacionalidad es mucho más manifiesta -por cierto- que la correspondiente a las precipitaciones (fig. 3). Así, los mayores registros de escorrentía se producen durante el invierno. Mientras que durante el verano, tanto la absorción de agua por parte del suelo - a la sazón con escasa humedad- como la interceptación -y posterior evaporación- de parte del agua de lluvia por la vegetación, llevan a importantes caídas del volumen de escorrentía a la salida de las cuencas.

\subsection{Descarga y concentración de sedimentos}

Con respecto a la generación de sedimentos, se observan significativas e interesantes diferencias entre las cuencas. 

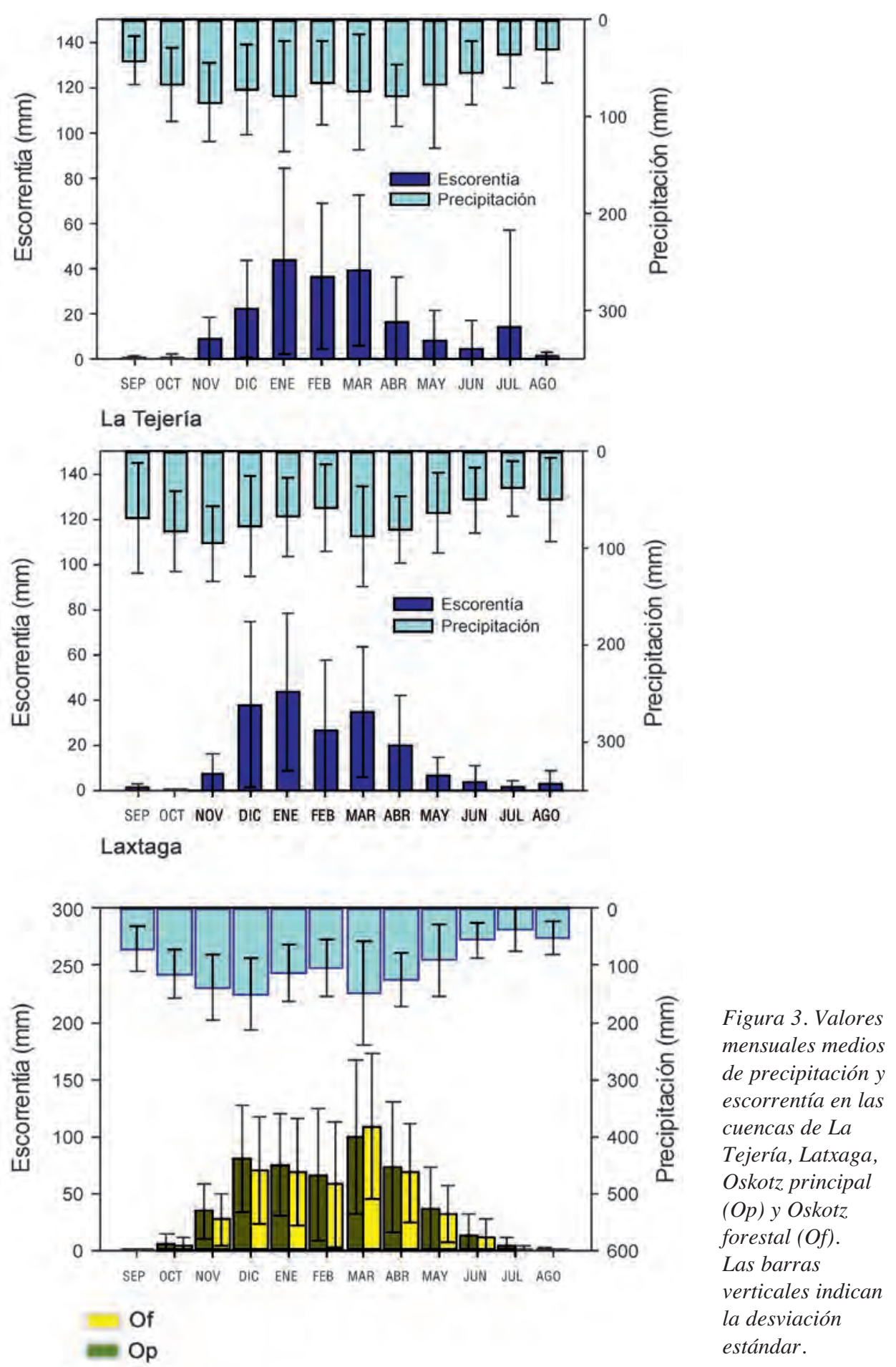
La Tejería presenta una descarga de sedimentos anual promedio (1833 kg/ ha año) alrededor de 4 veces mayor que la registrada en Latxaga (457 kg/ ha año) (fig. 2); igualmente, las diferencias en concentración anual media de sedimentos en las cuencas cerealistas es también notoria: $383 \mathrm{mg} / \mathrm{l}$ y $128 \mathrm{mg} / \mathrm{l}$, respectivamente. Con respecto a la cuenca de Oskotz, tanto la descarga anual media de sedimentos (Op: $641 \mathrm{~kg} / \mathrm{ha}$ año; Of: $770 \mathrm{~kg} / \mathrm{ha}$ año; fig. 2) como la concentración de los mismos (Op: 49 mg/l; Of: 65 mg/l) fueron mayores en Of; y en general inferiores a las cerealistas, al menos que las correspondientes a La Tejería. Cabe destacar que, en todos los casos citados, la variabilidad interanual de descarga/concentración de sedimentos es importante puesto que estos parámetros están básicamente controlados por los eventos de lluvias; de por sí, precisamente, altamente variables en nuestro clima.

Por otro lado, la descarga de sedimentos en todas las cuencas presenta una clara estacionalidad (fig. 4), con los mayores registros en invierno y principios de primavera; curiosamente en coincidencia con la ocurrencia de las precipitaciones de menor poder erosivo (ver arriba). La explicación más plausible, sería la siguiente. Durante el invierno, los suelos se encuentran mayormente desprotegidos -tanto aquellos bajo cereales como bajo bosques caducifolios- y por tanto vulnerables a la erosión. Además, la alta humedad edáfica durante el invierno favorece la generación de escorrentía superficial, incrementando -aún más- los procesos erosivos. Por otro lado, las frecuentemente erosivas lluvias estivales son, en alguna medida, atenuadas por la cubierta vegetal o sus restos. Igualmente, durante el verano, como ya se indicó, gran parte del agua caída es absorbida por el suelo -a la sazón- con escaso contenido hídrico. Por ende, se puede afirmar que la descarga de sedimentos en las cuencas está principalmente controlada por las condiciones del suelo, más que por el poder erosivo de las lluvias, per se. Aun así, gran parte de la descarga de sedimentos a la salida de las diferentes cuencas se asocia a sólo un reducido número de eventos de lluvia; hecho que concuerda con estudios y observaciones realizados en Navarra, con anterioridad (Donézar et al., 1990a, b; Casalí et al., 1999).

Por otra parte, la monitorización de surcos y cárcavas efímeras realizadas en ambas cuencas cerealistas en el período 2003/2004 (De Santisteban et. al, 2005) -pero continuada hasta el presente, aunque de forma ocular- evidencia una gran variabilidad espacial en cuanto al fenómeno erosivo. Es así que, en algunas pequeñas subcuencas de La Tejería se registraron pérdidas de suelos hasta un orden de magnitud mayores que el promedio de toda la cuenca; esto debido a la notoria erosión por flujos concentrados (surcos y cárcavas efímeras) detectada en estas subcuencas. Por el contrario, no se detecta ninguna forma de erosión por flujos concentrados ni movimientos de masa en la cuenca de Latxaga.

Las marcadas diferencias en valores de erosión (descarga de sedimento) entre las cuencas cerealistas podrían atribuirse, en parte, a la topografía predominante en cada una de estas cuencas como también a la diferente pendiente general del cauce principal. En efecto, la cuenca de La Tejería presenta una forma circular (fig. 1) -según lo indican los valores que toma el índice de Gravelius (Bendjoudyand Hubert, 2002) y el Factor de Forma (Monsalve Sáenz, 1999) (Tabla 1) - y una topografía poco abrupta, además de una elevada pendiente general de sus cauces; todo lo cual facilita la generación de caudales elevados. Igualmente, los taludes de los cauces principales de La Tejería son más vulne- 

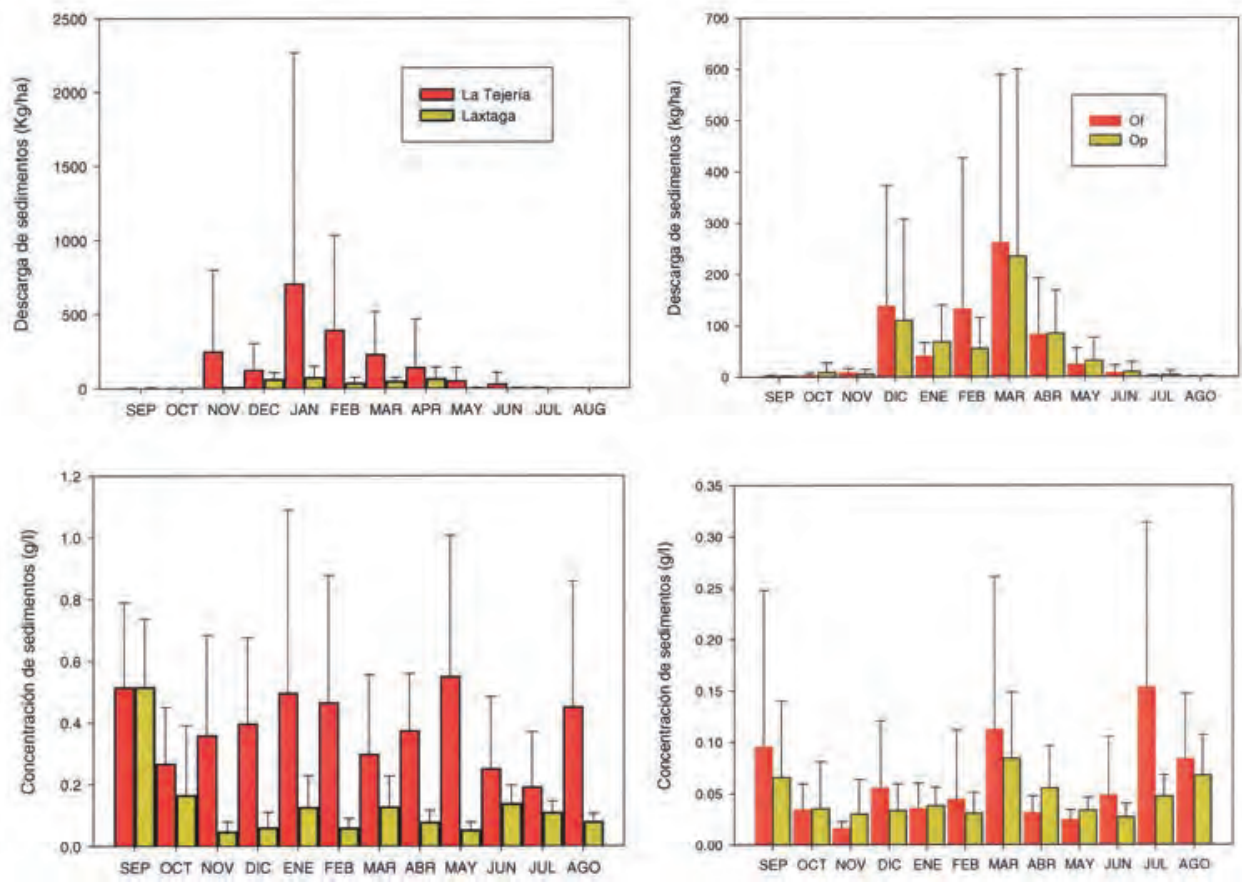

Figura 4. Valores mensuales medios de descarga y concentración de sedimentos en las cuencas de La Tejería, Latxaga, Oskotz principal (Op) y Oskotz forestal (Of). Las barras verticales indican la desviación estándar.

rables a la acción erosiva de la corriente al estar casi desprovistos de vegetación. Por otro lado, en La Tejería se registran importantes deslizamientos de tierra que aportan sedimentos a los cauces; como el acaecido en 2001 y 2003 que afectó a una superficie de 1.7 ha (De Santisteban et al., 2005). En cambio, en la cuenca de Latxaga, la topografía es marcadamente más abrupta y la forma menos circular (fig. 1), la pendiente general de los cauces es menor y éstos se encuentran cubiertos de vegetación; lo cual, además, favorece la sedimentación del material arrancado de las laderas. Por último, en Latxaga no se registran deslizamientos de tierras de importancia que contribuyan a la descarga de sedimentos.

En la cuenca de Oskotz, por su parte, la protección de los suelos contra el efecto erosivo de las lluvias, dado por la presencia de pastos o bosques, minimiza la erosión. Esto es así a pesar de registrarse, en esta cuenca, -como ya se indicó- valores de precipitación y escorrentía muy superiores a los que se producen en las cuencas cerealistas. Sin embargo, en la subcuenca forestal (Of), pueden medirse intensidades de erosión elevadas en terrenos que quedaron desprotegidos luego de una tala (aclareo). Un ejemplo de esto lo constituyen los registros de exportación de sedimentos para el período 2002/ 2003 (datos no mostrados) donde la intensidad de erosión de suelos en Of superó ampliamente a la registrada aún en la cuenca cerealista de Latxaga. Incluso, Op superó a Latxaga en valores de intensidad de erosión para el período mencionado; recuérdese 
que más de la mitad de la superficie de Op está ocupada por bosques. Más aún, y como se menciona anteriormente, en la cuenca de Oskotz la mayor parte de la exportación de sedimentos ocurre en invierno. Sin embargo, se han registrado también valores significativos en los meses de junio y julio, debido, probablemente, a talas selectivas que dejaron el suelo desprotegido frente a aisladas pero importantes precipitaciones.

\subsection{Exportación de nitratos y fosfatos}

La descarga anual de nitratos por escorrentía en La Tejería y Latxaga es de aproximadamente $132 \mathrm{~kg} / \mathrm{ha}$ año y $48 \mathrm{~kg} / \mathrm{ha}$ año, respectivamente (fig. 2) lo que significaría una pérdida del $22 \%$ y $10 \%$ del total del nitrógeno aplicado a través del abonado. Curiosamente, esto último es así, a pesar de que, como ya se dijo, la cuenca de Latxaga habría recibido una mayor cantidad de fertilizantes nitrogenados que La Tejería. Así, estas pérdidas son, en general, elevadas si la comparamos con valores registrados en otros estudios. De esta manera, el límite legal para agua potable (50 mg/l de NO3) es superado ampliamente en La Tejería, en todos sus registros; aumentando, además, los riesgos de eutrofización del ecosistema. Sin embargo, en Latxaga, este umbral sólo se excede esporádicamente.

Tanto la descarga como la concentración de nitratos muestran una clara variación estacional en las dos cuencas cerealistas; con los mayores registros durante el invierno y principio de primavera (fig. 5). Esto, puede ser en gran parte debido a la menor absorción biótica de nitrógeno durante la época de dormancia; siendo así más factible las pérdidas de nitratos por escorrentía (Campbell et al., 2004). Además, la escorrentía es, como se indicó, máxima durante el invierno. Con todo, la variabilidad de la concentración de nitratos es relativamente pequeña. Esto sería así debido a que la aportación de nitratos no sólo está afectada por la precipitación, sino también por el flujo base de las cuencas. De tal manera que este segundo aporte (por aguas subterráneas) actuaría como amortiguador de los bruscos cambios de concentración de nitratos generados por el carácter errático -aunque estacional- de las lluvias.

Por su parte, la concentración y descarga de fosfatos también es superior -aunque ligeramente- en La Tejería que en Latxaga (fig. 2). Pero con registros sujetos, en ambas cuencas, a una elevada variabilidad. Estos valores, también en estas dos cuencas, corresponden a aguas con un riesgo significativo de eutrofización (EEA, 1999). Esto, a pesar de la elevada fijación de fosfatos que se produce en estos suelos debido a la presencia de altas cantidades de carbonatos (Tisdale and Nelson, 1966). Igualmente, la descarga de fosfato muestra una cierta estacionalidad (fig. 6) en sus registros, no así la concentración del mismo, que no parece responder a una variación sistemática (fig. 6).

Las diferencias en descarga/concentración tanto de nitratos como de fosfatos entre las cuencas cerealistas podrían, además, en parte atribuirse al hecho de que Latxaga presenta una red de drenaje más amplia y densamente vegetada que su par La Tejería. Los beneficios de estos buffers dados por la vegetación son ampliamente reconocidos (por ej. Klapproth and Johnson, 2000; Broadmeadow and Niesbet, 2004; Fiener and Auerswald, 2006). 

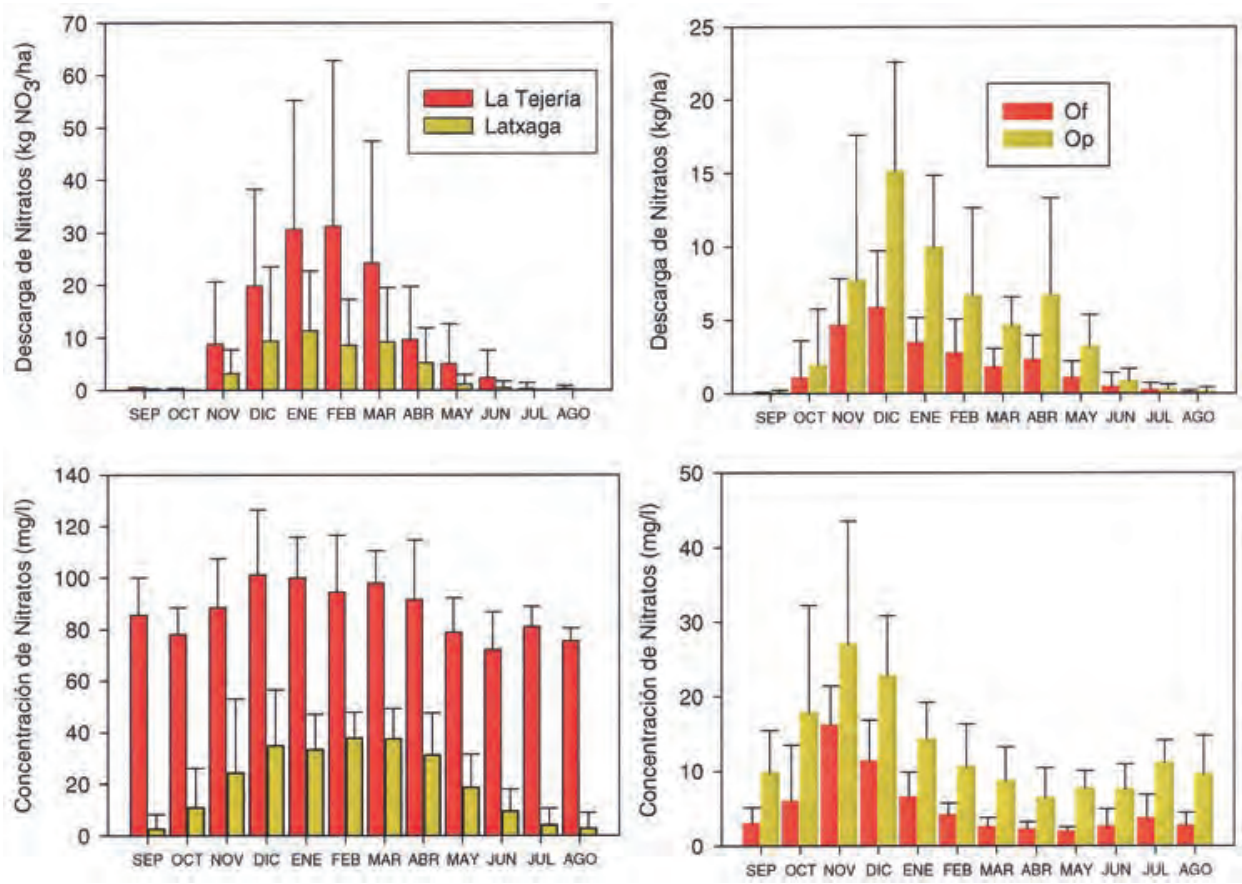

Figura 5. Valores mensuales medios de descarga y concentración de nitratos en las cuencas de La Tejería, Latxaga, Oskotz principal (Op) y Oskotz forestal (Of). Las barras verticales indican la desviación estándar.

Por su parte, en la cuenca de Oskotz, la concentración y descarga de nitratos no alcanza valores tan elevados como en las cuencas cerealistas (fig. 2). La descarga anual media de nitratos en Op (54 kg/ha año) duplica a la registrada en Of (22 kg /ha año); con similares contrastes en cuanto a la concentración de nitratos: 33 mg/l y 14 mg/l, respectivamente. Estas diferencias están en relación con la elevada actividad ganadera (vertido de purines) y agrícola en Op. Además, los suelos bajo bosque -por su alto poder denitrificante- reducen el aporte de nitrógeno hacia las corrientes de agua (Hayakawa et al., 2006). Igualmente, en cuencas dominadas por pasturas suelen registrarse exportaciones de nitrógeno que duplican a las medidas en cuencas forestales (Álvarez-Cobelas et al., 2008). Por último en relación al nitrógeno, se puede afirmar -en términos generales- que la descarga total del mismo en Oskotz (Op/Of) es elevada en comparación a similares cuencas descriptas en la literatura, a nivel mundial (p.ej., Frink, 1991; Campbell et al., 2004; Álvarez-Cobelas et al., 2008).

Por otro lado, la descarga de fosfato en Oskotz (Op/Of) resulta marcadamente superior a la observada en ambas cuencas cerealistas (fig. 2). Además, la descarga/ concentración anual media de fosfato en Op $(0.76 \mathrm{~kg} / \mathrm{ha}$ año; $0,44 \mathrm{mg} / \mathrm{l})$ duplica a la correspondiente a Of $(0.36 \mathrm{~kg} / \mathrm{ha}$ año; $0.19 \mathrm{mg} / \mathrm{l})$; resultando así las aguas de esta cuenca -aun en la subcuenca forestal, Of- con serios riesgos de eutrofización (EEA, 1999). 

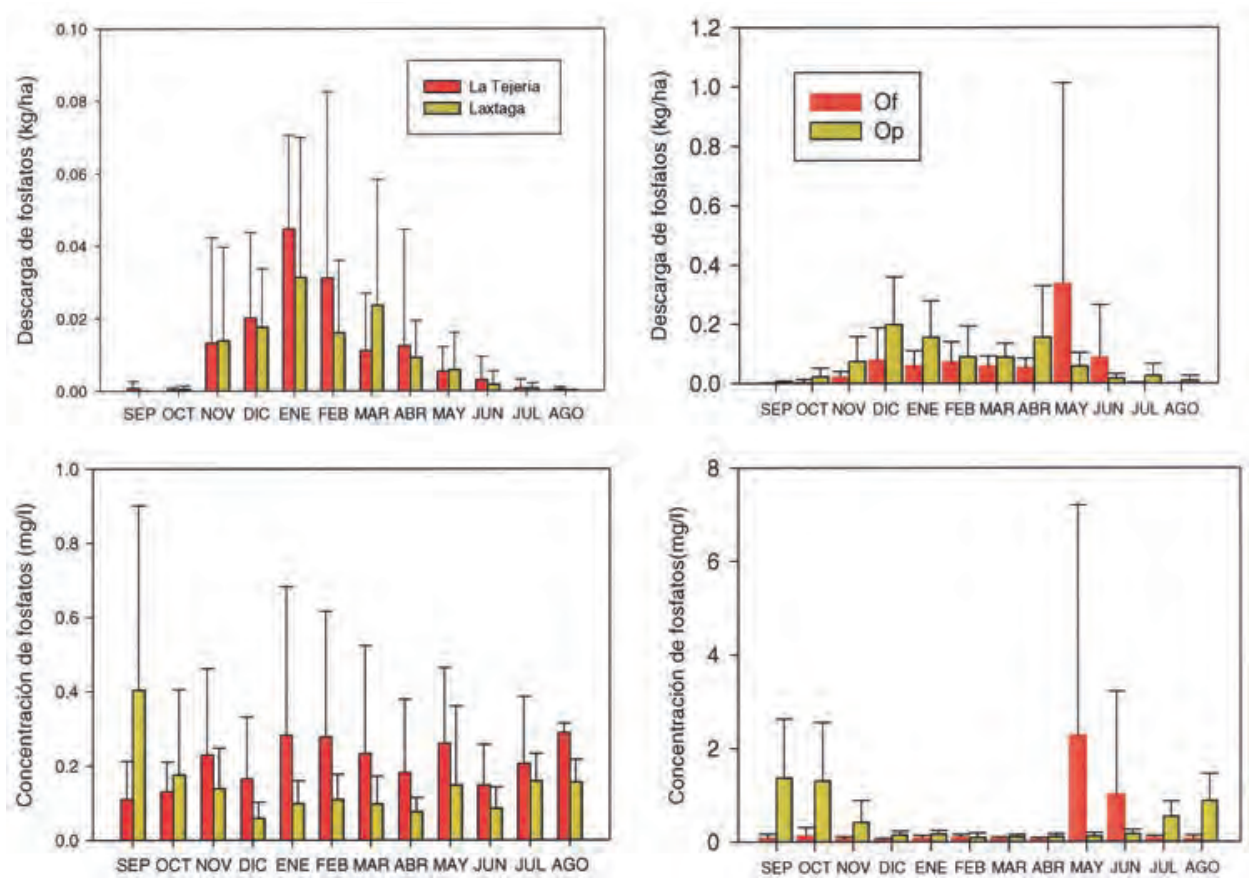

Figura 6. Valores mensuales medios de descarga y concentración de fosfatos en las cuencas de La Tejería, Latxaga, Oskotz principal (Op) y Oskotz forestal (Of). Las barras verticales indican la desviación estándar.

La menor fijación de fosfatos en los suelos de Oskotz, explica la mayor concentración de los mismos en el agua de escorrentía que las encontradas en las aguas de las cuencas cerealistas (fig. 2); estas últimas con suelos predominantemente alcalinos ( $\mathrm{pH}>8$ ) y por tanto propensos a la inmovilización del fósforo, como se destacó con anterioridad.

Además, la actividad ganadera lleva a que Op registre concentraciones de fosfatos -proveniente del estiércol y purines- disuelto en el agua de escorrentía, mayores a las encontradas en Of. De ahí que las menores concentraciones de fosfato en Op se observan en el invierno, en coincidencia precisamente con los mayores volúmenes de escorrentía -y por tanto, de dilución- (fig. 6). Por el contrario, no se registra una clara variación estacional del fosfato en Of.

Los elevados registros de fósforo en Oskotz resultan preocupantes, pues si en las cuencas cerealistas se alcanzan ya valores críticos de fósforo -a pesar de la significativa reducción de fosfatos por fijación- el riesgo de eutrofización sería aún mayor en Oskotz, sobre todo en Op. Precisamente, en la estación hidrológica de Op, se observan, con frecuencia, importantes poblaciones de algas. No obstante, los valores de exportación de fósforo en Oskotz no pueden considerarse extremos cuando se comparan con los registrados en cuencas similares, a nivel mundial (p.ej., Dilon and Kirchner, 1975; Frink, 1991). 


\section{Conclusiones}

En este estudio se describen en detalle el comportamiento hidrológico-erosivo de típicas cuencas agrarias de Navarra, a lo largo de varios lustros de continuo registro de datos (precipitación/escorrentía; sedimentos, nitratos y fosfatos evacuados). En concreto, se analizan dos cuencas cerealistas y una cuenca forestal/pastoril; esta última con una subcuenca forestal, en su casi totalidad.

Existe una elevada variabilidad interanual en cuanto a intensidad de erosión y exportación de nutrientes en las cuencas en estudio; lo cual es propio de la naturaleza de nuestro clima. Por otro lado, y para un mismo período de tiempo, cada una de las cuencas presenta, además, un comportamiento muy diferente con respecto a las variables citadas. Llama la atención que cuencas cerealistas con clima y suelos similares registren intensidades de erosión muy diferentes. Es así que, en la cuenca de La Tejería se han detectado elevadas intensidades de erosión, las cuales, eventualmente, estarían por encima de los niveles de tolerancia. En Latxaga, en cambio, los registros de erosión difícilmente alcanzan valores preocupantes. De esto concluimos que tanto la vegetación presente en los cauces, como la morfología y topografía propias de las cuencas, juegan un papel crucial en el fenómeno de la erosión, muy superior al que, inicialmente, podría pensarse. Es asimismo destacable y llamativo que, en cuencas mayoritariamente forestales como lo es Oskotz, se registren intensidades de erosión similares a las de una cuenca cerealista como Latxaga. No debemos sobrestimar a las áreas boscosas en su papel protector del suelo ante el proceso erosivo, ya que un inadecuado manejo forestal puede dar lugar a pérdidas de suelo aún superiores a las registradas en terrenos totalmente cultivados.

La calidad del agua de nuestras cuencas (y embalses) está siendo afectada por la intensa actividad agrícola y ganadera, a través de las elevadas cantidades de nitratos y fosfatos provenientes de fertilizantes y purines. Una de las cuencas cerealistas (La Tejería) presenta, de forma permanente, una concentración de nitratos superior a los límites tolerables. Además de registrarse, en ambas cuencas cerealistas, pérdidas significativas del abonado nitrogenado aportado.

En todas las cuencas se midieron concentraciones de fosfato superiores a valores críticos para la eutrofización. Esto último se observa claramente en la cuenca de Oskotz debido a la intensa actividad ganadera. La gran capacidad de fijación de fosfatos de suelos calcáreos asociados a las cuencas cerealistas resulta insuficiente para reducir los fosfatos solubles a valores discretos. Las implicancias de este problema no sólo son las obvias, en cuanto a que se ve afectada el agua destinada a consumo humano, sino también otras igualmente serias. Nos referimos a la degradación de ecosistemas, especialmente acuáticos, adyacentes a las fuentes de polución.

Estas acusadas diferencias ponen de manifiesto, una vez más, la riqueza y diversidad de nuestros paisajes, clima, suelos, y geología. A pesar de la necesidad de un aún mayor número de años con registros de datos, se considera que la información aportada en este trabajo es de gran valor e interés para los estudios relacionados con el efecto de la actividad agraria en cuencas de Navarra -y similares en España- en el medio ambien- 
te. Por último, para un estudio más acabado sobre nuestras cuencas sería importante contar con iguales estudios a los realizados, pero ahora en cuencas inalteradas, de tal manera de evaluar fehacientemente el efecto antrópico en el medio natural.

\section{Agradecimientos}

Estos estudios fueron parcialmente financiados a través de los Proyectos I+D CGL2007-63453 y CGL2011-24336 pertenecientes al Ministerio de Ciencia e Innovación del Gobierno de España. Los autores agradecen, además, la generosa contribución del Departamento de Desarrollo Rural y Medio Ambiente del Gobierno de Navarra.

\section{Referencias bibliográficas}

Álvarez-Cobelas, M., Angeler, D. G., SÁnchez-CArrillo, S. (2008). Export of nitrogen from catchments: A worldwide analysis. Environmental Pollution, 156: 261-269.

Bendjoudi, H., Hubert, P. (2002). Le coefficient de compacité de Gravelius: analyse critique d'un indice de forme des bassins versants. Hydrological Sciences Journal, 47 (6): 921-930.

Boix-Fayos, C., Martínez-Mena, M., Arnau-Rosalén, E., Calvo-Cases, A., CastiLlo, V., Albaladejo, J. (2006). Measuring soil erosion by field plots: Understanding the sources of variation. Earth-Science Reviews, 78: 267-285.

Broadmeadow, S., NiEsbet, T. R. (2004). The effects of riparian forest management on the freshwater environment: a literature review of best management practice. Hydrology and Earth System Sciences, 8(3): 286-305.

Campbell, J. L., Hornbeck, J. W., Mitchell, M. J., Adams, M. B., Castro, M. S., DrisColl, C. T., Kahl, J. S., Kochenderfer, J. N., Likens, G. E., Lynch, J. A., MurDOCH, P. S., NELSON, S. J., SHANLEY, J. B. (2004). Input-output budgets of inorganic nitrogen for 24 forest watersheds in the Northeastern United States: A review. Water, Air, and Soil Pollution, 151: 373-396.

CAsalí, J., LóPez, J. J., GiRÁldez, J. V. (1999). Ephemeral gully erosion in southern Navarra (Spain). Catena, 36: 65-84.

Casalí, J., Gastesi, R., Álvarez-Mozos, J., De Santisteban, L. M., Lersundi, J. D. V., Giménez, R., Larrañaga, A., GoÑi, M., Agirre, U., Campo, M. A., López, J. J., DONEZAR, M. (2008). Runoff, erosion, and water quality of agricultural watersheds in central Navarre (Spain). Agricultural Water Management, 95: 1111-1128.

Casalí, J., Giménez, R., Díez, J., Álvarez-Mozos, J., Del V alle de Lersundi, J., GoÑI, M., CAMPo, M. A., Chahor, Y., GASTESI, R., LóPez, J. (2010). Sediment production and water quality of watersheds with contrasting land use in Navarre (Spain). Agricultural Water Management, 97: 1683-1694. 
De Santisteban, L., Campo, M. A., Casalí, J. (2005). Fuentes de sedimentos en cuencas cultivadas de Navarra. En: III Congreso de Agroingeniería (Aguado, P. J., Juan, A., Morán, J. M., eds). León, Spain, pp. 341-232.

De Santisteban, L. M., Casalí, J., López, J. J. (2006). Assessing soil erosion rates in cultivated areas of Navarre (Spain). Earth Surface Processes and Landforms, 31: 487-506.

Dillon, P. J., Kirchner, W. B. (1975). The effects of geology and land use on the export of phosphorus from watersheds. Water Research, 9: 135-148.

Donézar, M., Illarregui, M., Del Val, J., Del Valle de Lersundi, J. (1990a). Erosión actual en Navarra. Gobierno de Navarra. Ministerio de Comercio y Turismo.

Donézar, M., Illarregui, M., Del Val, J., Del Valle de Lersundi, J. (1990b). Erosión potencial en Navarra. Gobierno de Navarra. Ministerio de Comercio y Turismo.

EEA (European Environment Agency) (1999). Nutrients in European Ecosystems. Environmental Assessment Report No. 4, EEA, Copenhagen.

Fiener, P., Auerswald, K. (2006). Seasonal variation of grassed waterway effectiveness in reducing runoff and sediment delivery from agricultural watersheds in temperate Europe. Soil \& Tillage Research, 87: 48-58.

FRINK, C. R. (1991). Estimating nutrient exports to estuaries. Journal of Environmental Quality, 20: 717-724.

García-Ruiz, J. M., LóPez Bermúdez, F. (2009). La erosión del suelo en España. Sociedad Española de Geomorfología. 441 pp.

Gobierno de NAVARRA (1997). Mapa geológico de Navarra 1:200.000. Gobierno de Navarra, Departamento de Obras Públicas, Transportes y Comunicaciones, Pamplona, Spain.

Gobierno de Navarra (2001). Estudio Agroclimático de Navarra (CD). Gobierno de Navarra, Departamento de Agricultura, Ganadería y Alimentación, Servicio de Estructuras Agrarias. Pamplona, Spain.

Hayakawa, A., Shimizu, M., Woli, K. P., Kuramochi, K., Hatano, R. (2006). Evaluating stream water quality through land use analysis in two grassland catchments: Impact of wetlands on stream nitrogen concentration. Journal of Environmental Quality, 35: 617-627.

IsIdORo, D., QuíleZ, D., AragüÉs, R. (2003). Sampling strategies for the estimation of salt and nitrate loads in irrigation return flows: La Violada Gully (Spain) as a case study. Journal of Hydrology, 271: 39-51.

Klapproth, J. C., Johnson, J. E. (2000). Understanding the science behind riparian forest buffers: effects on water quality. Publication 420-151. Virginia Cooperative Extension, Petersburg. 
Llamas, J. (1993). Hidrología General. Principios y Aplicaciones. Servicio Editorial de la Universidad del País Vasco, Bilbao.

Monsalve SÁenz, G. (1999). Hidrología en la ingeniería. Alfaomega. $2^{a}$ edición. 358 pp.

Morgan, R. P. C. (2005). Soil erosion and conservation. Blackwell Publishing. $3^{\text {a }}$ edición. 304 pp.

Tisdale, S., Nelson, W. (1966). Soil Fertility and Fertilizers. The MacMillan Company. New York. $2^{\text {a }}$ edición. $752 \mathrm{pp}$. 